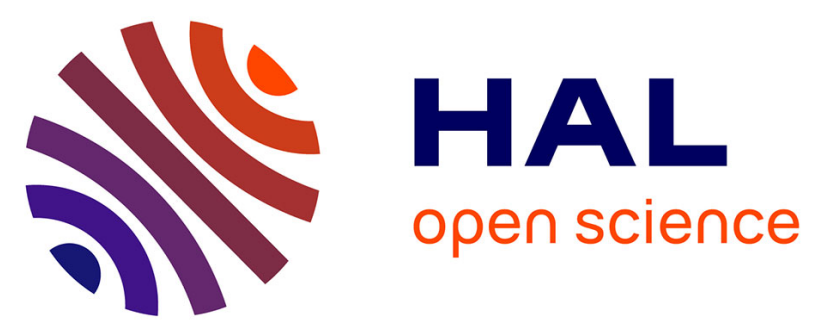

\title{
Coherent dynamics of resonantly excited excitons in monolayers of transition metal dichalcogenides
}

Tomasz Jakubczyk, Miroslav Bartos, Lorenzo Scarpelli, Karol Nogajewski, Wolfgang Langbein, Marek Potemski, Jacek Kasprzak

\section{- To cite this version:}

Tomasz Jakubczyk, Miroslav Bartos, Lorenzo Scarpelli, Karol Nogajewski, Wolfgang Langbein, et al.. Coherent dynamics of resonantly excited excitons in monolayers of transition metal dichalcogenides. Ultrafast Phenomena and Nanophotonics XXIV, Feb 2020, San Francisco, United States. pp.47, 10.1117/12.2549229 . hal-03012880

\section{HAL Id: hal-03012880 https://hal.science/hal-03012880}

Submitted on 18 Nov 2020

HAL is a multi-disciplinary open access archive for the deposit and dissemination of scientific research documents, whether they are published or not. The documents may come from teaching and research institutions in France or abroad, or from public or private research centers.
L'archive ouverte pluridisciplinaire HAL, est destinée au dépôt et à la diffusion de documents scientifiques de niveau recherche, publiés ou non, émanant des établissements d'enseignement et de recherche français ou étrangers, des laboratoires publics ou privés. 
Return to the Manage Active Submissions page at http://spie.org/submissions/tasks.aspx and approve or disapprove this submission. Your manuscript will not be published without this approval. Please contact author_help@spie.org with any questions or concerns.

\title{
Coherent dynamics of resonantly excited excitons in monolayers of transition metal dichalcogenides
}

\author{
Tomasz Jakubczyk ${ }^{a b}$, Miroslav Bartos ${ }^{c d}$, Lorenzo Scarpelli ${ }^{e}$, Karol Nogajewski ${ }^{c f}$, \\ Wolfgang Langbein ${ }^{g}$, Marek Potemski ${ }^{c f}$ and Jacek Kasprzak ${ }^{a}$
}

${ }^{a}$ Univ. Grenoble Alpes, CNRS, Grenoble INP, Institut Néel, 38000 Grenoble, France

${ }^{b}$ Department of Physics, University of Basel, 4056 Basel, Switzerland

${ }^{c}$ Laboratoire National des Champs Magnétiques Intenses, CNRS-UGA-UPS-INSA-EMFL, 25

Av. des Martyrs, 38042 Grenoble, France

${ }^{d}$ Central European Institute of Technology, Brno University of Technology, Purkynova 656/123, 61200 Brno, Czech Republic

${ }^{e}$ ARC Centre of Excellence for Engineered Quantum Systems, Macquarie University, Sydney, New South Wales, Australia

${ }^{f}$ Faculty of Physics, University of Warsaw, ul. Pasteura 5, 02-093 Warszawa, Poland ${ }^{g}$ School of Physics and Astronomy, Cardiff University, The Parade, Cardiff CF24 3AA, UK

\begin{abstract}
We investigate dynamics of resonantly excited excitons in single-layers of $\mathrm{MoSe}_{2}$ and $\mathrm{WS}_{2}$ down to $4.5 \mathrm{~K}$. To this end, we measure the delay dependence of the heterodyne four-wave mixing (FWM) amplitude induced by three, short laser pulses. This signal depends not only on the population of optically active excitons, which affects the absorption of the probe, but also on the population of optically inactive states, by interaction-induced energy shift, influencing the refractive index experienced by the probe. As such, it offers insight into density dynamics of excitons which do not directly couple to photons. Reproducing the coherent signal detected in amplitude and phase, the FWM delay dependence is modeled by a coherent superposition of several exponential decay components, with characteristic time constants from 0.1 picosecond up to 1 nanosecond. With increasing excitation intensity and/or temperature, we observe strong interference effects in the FWM field amplitude, resulting in progressively more complex and nonintuitive signal dynamics. We attribute this behaviour to increasingly populated exciton dark states, which change the FWM field phase by the relative effect on absorption and refractive index. We observe that exciton recombination occurs on a significantly longer timescale in $\mathrm{WS}_{2}$ with respect to $\mathrm{MoSe}_{2}$, which is attributed to the dark character of exciton ground state in the former and the bright in the latter.
\end{abstract}

Keywords: coherent nonlinear spectroscopy, transition metal dichalcogenides, exciton dynamics four-wave mixing micro-spectroscopy

\section{INTRODUCTION}

Carrier relaxation dynamics in semiconductor devices, for example in optical amplifiers, is a relevant issue in modern optoelectronics and has been intensively studied using heterodyne pump-probe spectroscopy.$^{1-4}$ Such methods of ultrafast coherent spectroscopy are also employed in fundamental studies of excitons (EX), bound complexes of electrons and holes, hosted by emerging material systems and their nanostructures, such as colloidal quantum dots,${ }^{5}$ nanoplatelets ${ }^{6}$ perovskites,${ }^{7}$ single-layers (SLs) of semiconducting transition metal dichalcogenides $^{8}$ (TMDs) and their van der Waals heterostructures. ${ }^{9}$

In the SL limit, TMDs become direct band gap materials, with the gap located at $\mathrm{K}^{+/-}$points of the hexagonal Brillouin zone. The optical response, even at room temperature, is dominated by EXs with a binding

Further author information: (Send correspondence to J.K.)

J.K.: E-mail: jacek.kasprzak@neel.cnrs.fr

11278 - 47 V. 2 (p.1 of 10) / Color: No / Format: Letter / Date: 2/10/2020 3:59:32 AM

SPIE USE: DB Check, Prod Check, Notes: 
Return to the Manage Active Submissions page at http://spie.org/submissions/tasks.aspx and approve or disapprove this submission. Your manuscript will not be published without this approval. Please contact author_help@spie.org with any questions or concerns.

energy of several hundreds meV. With such a high binding energy - stemming from the two-dimensional confinement, reduced dielectric screening and high carrier effective masses - light-matter interaction is expected to be enhanced with respect to conventional semiconductors, like GaAs. This is experimentally confirmed by a strong excitonic absorption in TMD SLs of around 10\%. Also, they accommodate circularly polarized EXs, where the helicity of the transition $(\sigma+/ \sigma-)$ is linked with the index $(+/-)$ of the $\mathrm{K}$ valley. Thus, apart from spin-allowed (bright) transitions, there are also spin-forbidden (dark) ones, between different valleys. EXs with the in-plane momentum beyond the light cone cannot recombine radiatively either and are also called dark. The multitude of available dark states and their various feeding channels,${ }^{10}$ as well as the energetic structure varying from material to material, is expected to strongly affect the EX dynamics in the family of TMDs.

Time-resolved photoluminescence experiments ${ }^{11-13}$ performed on TMD SLs have shown bi-exponential or multi-exponential decays on a timescale of a few hundred picosecond. Owing to non-resonant excitation, the modeling and interpretation of photoluminescence is blurred due to the numerous intermediate states in the scattering pathway from the initially excited electron-hole pairs to the emission of the bright EX states. Conversely, resonant driving with a short laser pulse, directly imprints a well controlled density of bright EXs within the light cone into a well defined valley $\left(\mathrm{K}^{+}, \mathrm{K}^{-}\right.$or both).

This can be conveniently accomplished by performing four-wave mixing (FWM) spectroscopy.$^{14}$ The latter was recently employed to investigate dynamics of resonantly driven EX population in $\mathrm{MoSe}_{2} \mathrm{SLs}{ }^{8}$ Considering the excitonic landscape in TMD SLs, these initial FWM studies have established several dominating processes in the EX density dynamics, like direct radiative recombination competing with scattering and evolution of density in dark state reservoirs (out of the light cone direct spin-allowed, direct spin-forbidden, indirect spin-allowed, and indirect spin-forbidden). These experiments were however performed only down to $77 \mathrm{~K}$ and measurements at lower temperatures down to $4.2 \mathrm{~K}$ have not been carried out. Recently, an example of EX population dynamics measured via FWM was reported and discussed in Ref. [9] using $\mathrm{MoS}_{2}$ heterostructure.

In this work, we study dynamics of resonantly excited EXs in bare SLs of $\mathrm{MoSe}_{2}$ and $\mathrm{WS}_{2}$ down to $4.5 \mathrm{~K}$. We present a comprehensive set of FWM measurements on a timescale from 0.1-1300 ps, combining excitation power $(P)$ and temperature $(T)$ dependencies. Increasing $P$ and/or $T$, the dark states become increasingly more populated, resulting in more and more complex FWM delay traces. In these experiments, we observe prominent interference effects in the FWM amplitude, revealing different scattering channels between several EX reservoirs. We also conclude that exciton relaxation occurs on a significantly longer timescale in $\mathrm{WS}_{2}$ with respect to $\mathrm{MoSe}_{2}$, which is tentatively attributed to dark character of the exciton ground state in $\mathrm{WS}_{2}$ and the bright in $\mathrm{MoSe}_{2}$. Following Ref. [8], the FWM amplitude versus delay is modeled by a coherent superposition of up to five components of complex exponential decays, generating highly non-intuitive delay traces, yet reproducing the ensemble of our measurements very well.

\section{EXPERIMENT}

\subsection{Samples}

We here use two kinds of TMD SLs: $\mathrm{MoSe}_{2}$ and $\mathrm{WS}_{2}$, the same as the ones as explored in Ref. [15] and Ref. [16], respectively. In both cases, the SLs were produced by means of polydimethylsiloxane-based exfoliation of bulk crystals, purchased from HQ Graphene, and transferred onto a Si substrate with a $90 \mathrm{~nm} \mathrm{SiO}_{2}$ surface layer and then inserted into an optical He-flow cryostat. In $\mathrm{MoSe}_{2}$, the lowest conduction band and the highest valence band have the same spin, therefore the EX with the lowest energy is bright (optically active). It is worth to note that, the next conduction band hosting an electron with the opposite spin, and thus contributing to the dark EX, lies only $1.5 \mathrm{meV}$ away, ${ }^{17}$ around twenty times closer than previous theoretical predictions. ${ }^{18}$ In $\mathrm{WS}_{2} \mathrm{SLs}$ though the configuration is inverse, i.e., the lowest conduction band and the highest valence band have opposite spin, therefore the lowest lying EX is dark. The next conduction band, sharing the same spin projection as the valence band, is around $30 \mathrm{meV}$ higher, producing the spin-allowed bright exciton. The corresponding energy diagrams for both materials are depicted in Fig. 1 a.

\section{8 - 47 V. 2 (p.2 of 10) / Color: No / Format: Letter / Date: 2/10/2020 3:59:32 AM}


Return to the Manage Active Submissions page at http://spie.org/submissions/tasks.aspx and approve or disapprove this submission. Your manuscript will not be published without this approval. Please contact author_help@spie.org with any questions or concerns.
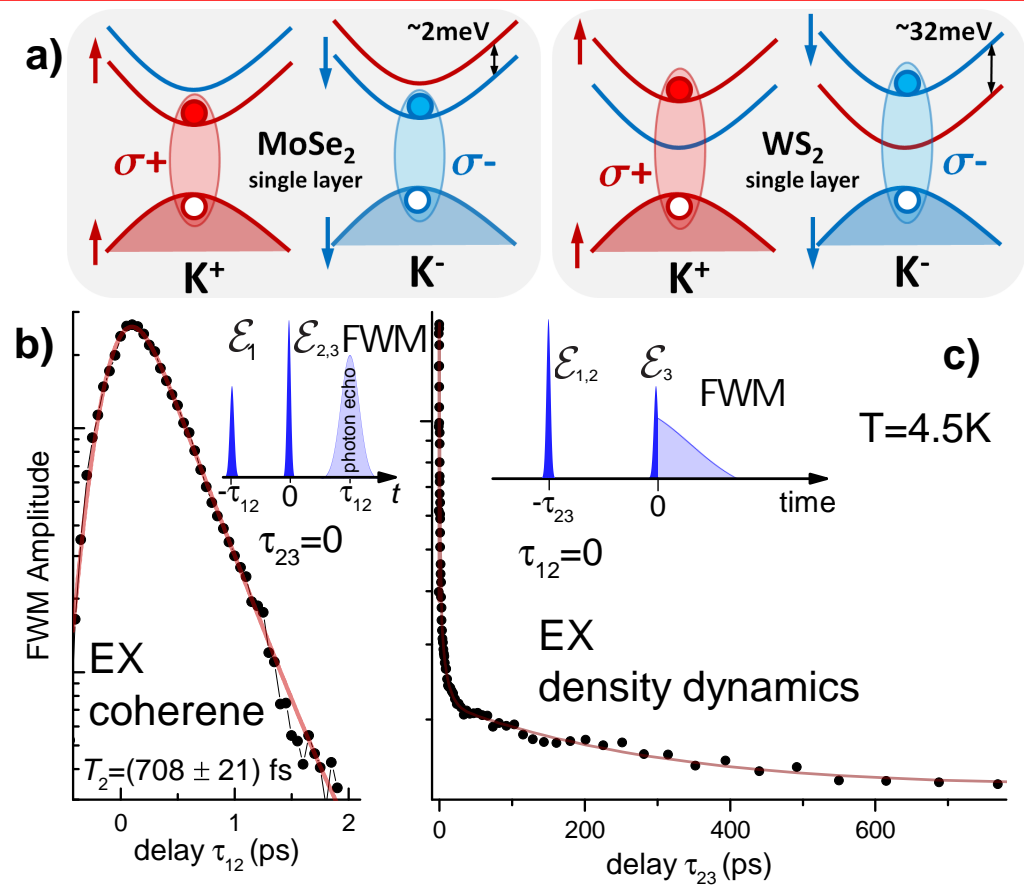

Figure 1. Direct exciton transitions in single-layers of transition metal dichalcogenides and their observables probed with four-wave mixing spectroscopy. a) Schema of band structure of TMD SLs, showing the highest lying valence band and the lowest lying pair of conduction bands, at the $\mathrm{K}^{+}$and $\mathrm{K}^{-}$points. Owing to the reverse energy splitting, in $\mathrm{MoSe}_{2}\left(\mathrm{WS}_{2}\right)$ the transition of the lowest energy is optically (in)active, bright (dark). b, c) Representative measurements on MoSe 2 SL at $\mathrm{T}=4.5 \mathrm{~K}$. b) Time-integrated FWM amplitude as a function of delay $\tau_{12}$, for $\tau_{23}=0$, as depicted in the inset, reflecting EX coherence dynamics. c) Time-integrated FWM amplitude as a function of delay $\tau_{23}$, for $\tau_{12}=0$, as depicted in the inset, reflecting EX population dynamics.

\subsection{Why four-wave mixing microscopy?}

SL-TMDs explored in these experiments have been fabricated as bare flakes deposited directly on $\mathrm{SiO}_{2} / \mathrm{Si}$ substrates. They are in direct contact with the environment, thus are prone to various microscopic and macroscopic disorder factors, such as: strain, wrinkling, flake deformations and cracks, lattice defects and vacancies, variation of charge state and of dielectric constant in the substrate. Their optical response is therefore affected by spectral inhomogeneous broadening ${ }^{15,19} \sigma$, which varies across the flake ${ }^{16}$ from nearly zero to a few tens of meV. When examining optical properties of TMDs it is desirable to use a spectroscopic tool capable to separate homogeneous and inhomogeneous contributions within the EX's spectral lineshape. In order to avoid adding up responses from areas exhibiting different optical properties, one would also preferentially employ microscopy, where diffraction limited excitation beams restrict spatial averaging to sub-micron areas.

The heterodyne FWM technique, exploited in this work, complies with these two requirements. FWM is a nonlinear polarization in the material, proportional to $\mu^{4} \mathcal{E}_{1}^{\star} \mathcal{E}_{2} \mathcal{E}_{3}$, where $\mu$ is the transition oscillator strength and $\mathcal{E}_{1,2,3}$ are three femto-second laser pulses. To study EXs in $\mathrm{MoSe}_{2} \mathrm{SL}$ at a light wavelength around $750 \mathrm{~nm}$, we use a Ti:Sapphire laser as pulse source (Tsunami-Femto, from Spectra-Physics). Conversely, for EXs in $\mathrm{WS}_{2}$ SL at around $590 \mathrm{~nm}$, we use optical parametric oscillator (Inspire, provided by Radiantis). We use a different set of optics adapted for these two wavelengths. The temporal chirp at the sample was pre-compensated using a grating-based pulseshaper to achieve Fourier-limited pulses.

FWM is here detected through optical heterodyning, enabling co-linear propagation of $\mathcal{E}_{1,2,3}$. This permits microscopy configuration, which has been required to measure coherent nonlinear response of individual excitons ${ }^{20-22}$ First, $\mathcal{E}_{1,2,3}$ are modulated by distinct radio frequencies $\Omega_{1,2,3}$ around $80 \mathrm{MHz}$ using acousto-optic deflectors. $\mathcal{E}_{1,2,3}$ propagate co-linearly and are focussed onto the sample down to the diffraction limit using a 
Return to the Manage Active Submissions page at http://spie.org/submissions/tasks.aspx and approve or disapprove this submission. Your manuscript will not be published without this approval. Please contact author_help@spie.org with any questions or concerns.

microscope objective of $\mathrm{NA}=0.65$. In the detection path, the reflected signal is interfered with the reference beam $\mathcal{E}_{\mathrm{R}}$ modulated at the phase-locked linear combination of radio-frequencies $\Omega_{3}+\Omega_{2}-\Omega_{1}$, picking up the FWM response from the reflection. Then, background-free interference between $\mathcal{E}_{\mathrm{R}}$ and FWM is recorded as non-oscillating spectral interference on a CCD camera, which is installed at the output of the imaging spectrometer. ${ }^{14}$ FWM amplitude and phase are obtained via spectral interferometry. Owing to the limited phase stability and lack of phase referencing ${ }^{23}$ in the current experiments, only the FWM amplitude is exploited.

As depicted in Fig. $1 \mathrm{~b}$, in a presence of $\sigma$, FWM forms a photon echo centered at $t=\tau_{12}$, where the latter is the delay between $\mathcal{E}_{1}$ and $\mathcal{E}_{2}$. The time-integrated (TI) FWM amplitude as a function of $\tau_{12}$ measures the EX coherence dynamics, which in the simple case decays exponentially. From the decay constant, one retrieves the microscopic dephasing time $T_{2}$, which can be translated to the full-with at half-maximum of the homogeneous broadening $\gamma=2 \hbar / T_{2}$. An example of such a measurement performed on $\mathrm{MoSe}_{2}$ at $\mathrm{T}=4.5 \mathrm{~K}$, yielding $T_{2} \simeq 0.7 \mathrm{ps,}$ is presented in Fig. $1 \mathrm{~b}$.

There are two principal channels causing EX dephasing: radiative decay (occuring in sub-ps range) and pure dephasing. The latter is due to the EX-EX scattering, such that the phase imprinted onto the EX cloud by $\mathcal{E}_{1,2,3}$ is rapidly lost. As a result the EX coherence withstands only a few ps. Instead, a part of EX density transferred to the dark states is present and evolves on a much longer timescale; up to at least $1 \mathrm{~ns}$, as presented in Fig. 1 c. To measure EX density dynamics we set $\tau_{12}=0 . \mathcal{E}_{1}$ and $\mathcal{E}_{2}$ induce EX density $\mathcal{E}_{1}^{\star} \mathcal{E}_{2}$, slowly oscillating at the frequency $\Omega_{2}-\Omega_{1}$. The EX density in different states affects via EX-EX interaction the EX polarization created by $\mathcal{E}_{3}$, inducing the detected FWM. The amplitude and phase of the FWM signal is therefore a probe of the EX-EX interaction between the optically probed transition and the EX states containing the density. To rephrase, after the delay $\tau_{23}$, the last pulse $\mathcal{E}_{3}$ arrives and triggers the FWM, which is proportional to the remaining EX density weighted by their mutual interactions. The dynamics of this combined effect is probed by reading out the TI-FWM amplitude versus $\tau_{23}$, as depicted in the inset of Fig. $1 \mathrm{c}$. The figure also shows that, at low $T$ and weak $P$, more than $90 \%$ of the EX population is gone already after only a few ps, owing to the radiative decay and simultaneous scattering to the EX dark states.

Another possible channel leading to the loss of density is the EX diffusion: during $\tau_{23}$, EXs can be ejected out of the sub- $\mu \mathrm{m}$ excitation spot. ${ }^{24}$ We have looked for such propagation by performing spatially-resolved FWM experiments. Namely, by spatially displacing (typically, by several $\mu \mathrm{m}$ ) $\mathcal{E}_{1,2}$ with respect to the probe beam $\mathcal{E}_{3}$ while detecting FWM, one becomes sensitive to the EX in-plane propagation effects. We have performed numerous experiments in such configurations, providing no evidence for EX diffusion in our samples. In fact, owing to the inhomogeneous broadening in these bare SLs, EXs are expected to be spatially localized and thus exhibiting nearly no diffusion at low temperatures.

The following of this work is devoted to presentation and modeling of such $\tau_{23}$-dependences measured at $\mathrm{MoSe}_{2}$ and $\mathrm{WS}_{2}$ for different $P$ and $T$.

\subsection{Modeling exciton density dynamics measured via heterodyne four-wave mixing.}

To describe the loss of EX density when increasing delay $\tau_{23}$, the proposed fitting function $R$ is a multi-exponential decay for $\tau_{23}>0$ and zero otherwise: $R\left(\tau_{23}\right) \propto \sum_{i=1}^{n} A_{i} \theta\left(\tau_{23}\right) \exp \left(\imath \phi_{i}-\frac{\tau_{23}}{\tau_{i}}\right)$. Because we employ a coherent detection scheme, it is sensitive to the phase of each of the decay processes. Therefore, $R$ is a complex function, providing characteristic times, amplitudes and phases of $n$ processes that are involved, $\left(\tau_{i}, A_{i}, \phi_{i}\right)$.

To mimic the experiments, the temporal form of the pulse train, employed to drive FWM, must be taken into account. Firstly, to take into account the finite pulse duration, $R$ is convoluted with a Gaussian characterized by a FWHM of $2 \sqrt{\ln (2)} \tau_{0}$ given by the laser autocorrelation. Secondly, owing to the lase repetition period $T_{R} \simeq 13 \mathrm{~ns}$, the signal might not fade away completely before the arrival of the following pulse sequence, but the leftover proportional to $\exp \left(-\frac{T_{R}}{\tau_{i}}\right)$ is present. When adding up such contribution from the second pulse sequence onward over an infinite number of pulses in the heterodyne detection scheme, the pile-up term is obtained as the geometric sum $\exp \left(-\frac{T_{R}}{\tau_{i}}\right)+\exp \left(-\frac{2 T_{R}}{\tau_{i}}\right)+\exp \left(-\frac{3 T_{R}}{\tau_{i}}\right)+\exp \left(-\frac{4 T_{R}}{\tau_{i}}\right)+\ldots=\frac{\exp \left(-\frac{T_{R}}{\tau_{i}}\right)}{1-\exp \left(-\frac{T_{R}}{\tau_{i}}\right)} \cdot R$ therefore takes the form: 
Return to the Manage Active Submissions page at http://spie.org/submissions/tasks.aspx and approve or disapprove this submission. Your manuscript will not be published without this approval. Please contact author_help@spie.org with any questions or concerns.
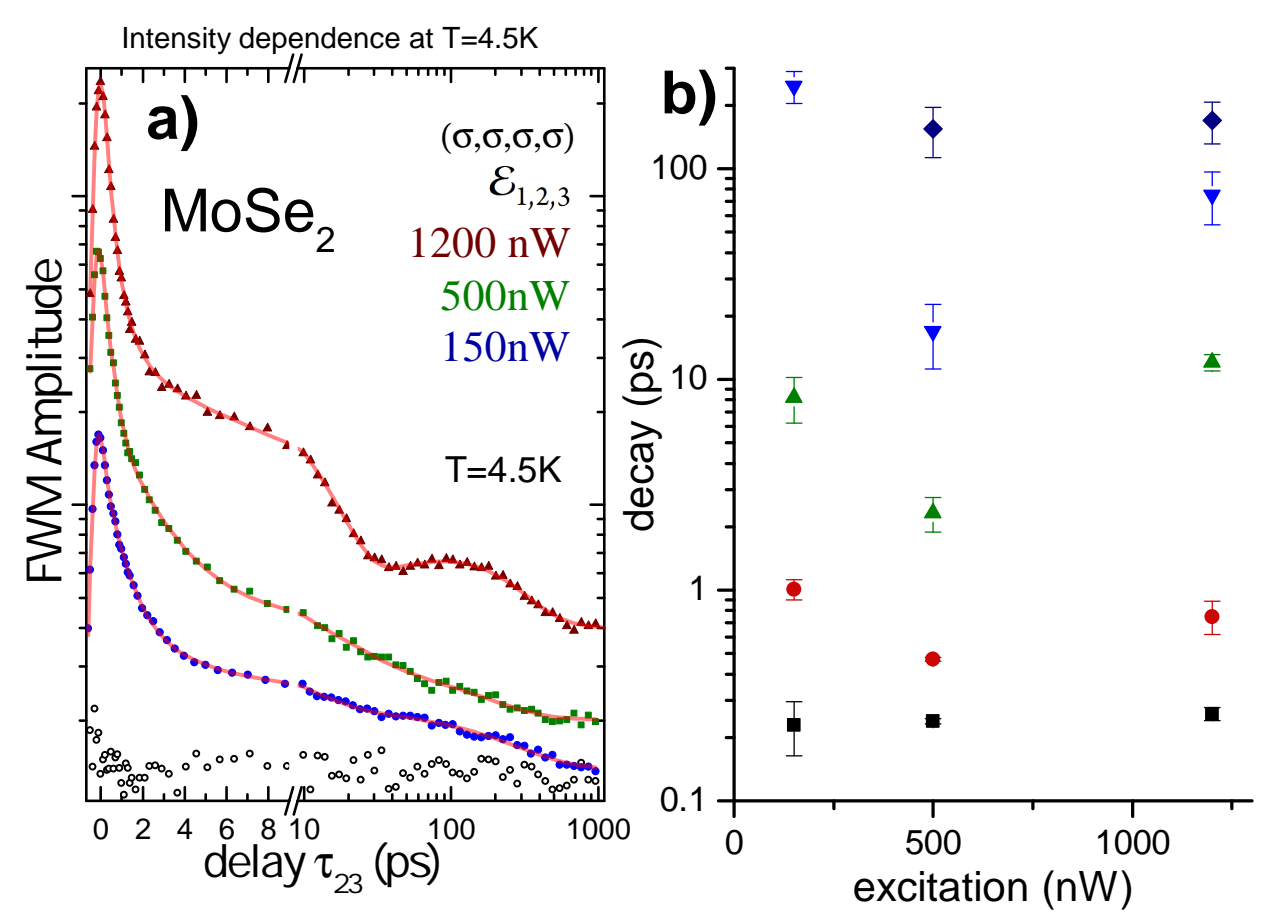

Figure 2. Exciton density dynamics in $\mathrm{MoSe}_{2}$ SL measured with four-wave mixing. Excitation power $P$ as indicated at $T=4.5 \mathrm{~K}$. For the highest $P=1200 \mathrm{nW}$ an oscillation observed between $10 \mathrm{ps}$ and $1000 \mathrm{ps}$ is due to the interference between different decay components, enabled by increased amplitude of the ones featuring longer decay constants. In all figures, fits are given with semi-transparent traces.

$$
R\left(\tau_{23}\right)=\sum_{i=1}^{n} A_{i}\left\{\left[\exp \left(\frac{T_{R}}{\tau_{i}}\right)-1\right]^{-1}+\frac{1}{2}\left[\left(1+\operatorname{erf}\left(\frac{\tau_{23}}{\tau_{0}}-\frac{\tau_{0}}{2 \tau_{i}}\right)\right]\right\} \times \exp \left(\imath \phi_{i}-\frac{\tau_{23}}{\tau_{i}}+\frac{\tau_{0}^{2}}{4 \tau_{i}^{2}}\right)\right.
$$

Finally, to fit the FWM amplitude we take absolute value of $R\left(\tau_{23}\right)$, namely $\left|R\left(\tau_{23}\right)\right|$, yielding a rather lengthy, yet still analytical form.

\section{RESULTS}

\section{1 $\mathrm{MoSe}_{2}$ single layer}

In Fig. 2 a we present the EX density dynamics measured on $\mathrm{MoSe}_{2} \mathrm{SL}$ at $T=4.5 \mathrm{~K}$ for three different driving powers $P$, as indicated. We estimate that exciting with $1 \mu \mathrm{W}$ generates around $10^{11} \mathrm{EX} / \mathrm{cm}^{2}$. Qualitatively, for the ensemble of our data we distinguish: i) signal at $\tau_{23}<0$ owing to the pile-up effect, ii) when approaching $\tau_{23}=0$ from negative values, we see a rise on a $100 \mathrm{fs}$ scale, due to the finite duration of the laser pulses, iii) strong, initial decay, by around an order of magnitude in the FWM amplitude over a few hundreds fs scale, attributed to the radiative decay and EX scattering to dark EX states. iv) followed after $\tau_{23}>1$ ps by a much longer decay, due to the population of the dark EX states and their subsequent relaxation and interaction with the bright ones, i.e., EXs within the light cone, obeying spin selection rules for radiative recombination.

The dynamics is fitted with $\left|R\left(\tau_{23}\right)\right|$ yielding several decay constants from $0.2 \mathrm{ps}$ to $200 \mathrm{ps}$, as reported in Fig. $2 \mathrm{~b}$. It is worth to point out that at the highest excitation power, after a dominating initial decay, for $\tau_{23}>10 \mathrm{ps}$ a strong modulation is measured and reproduced by the model. This is due to the interference between signals of different decay processes, whose phases are given by the relative effect on absorption and 
Return to the Manage Active Submissions page at http://spie.org/submissions/tasks.aspx and approve or disapprove this submission. Your manuscript will not be published without this approval. Please contact author_help@spie.org with any questions or concerns.

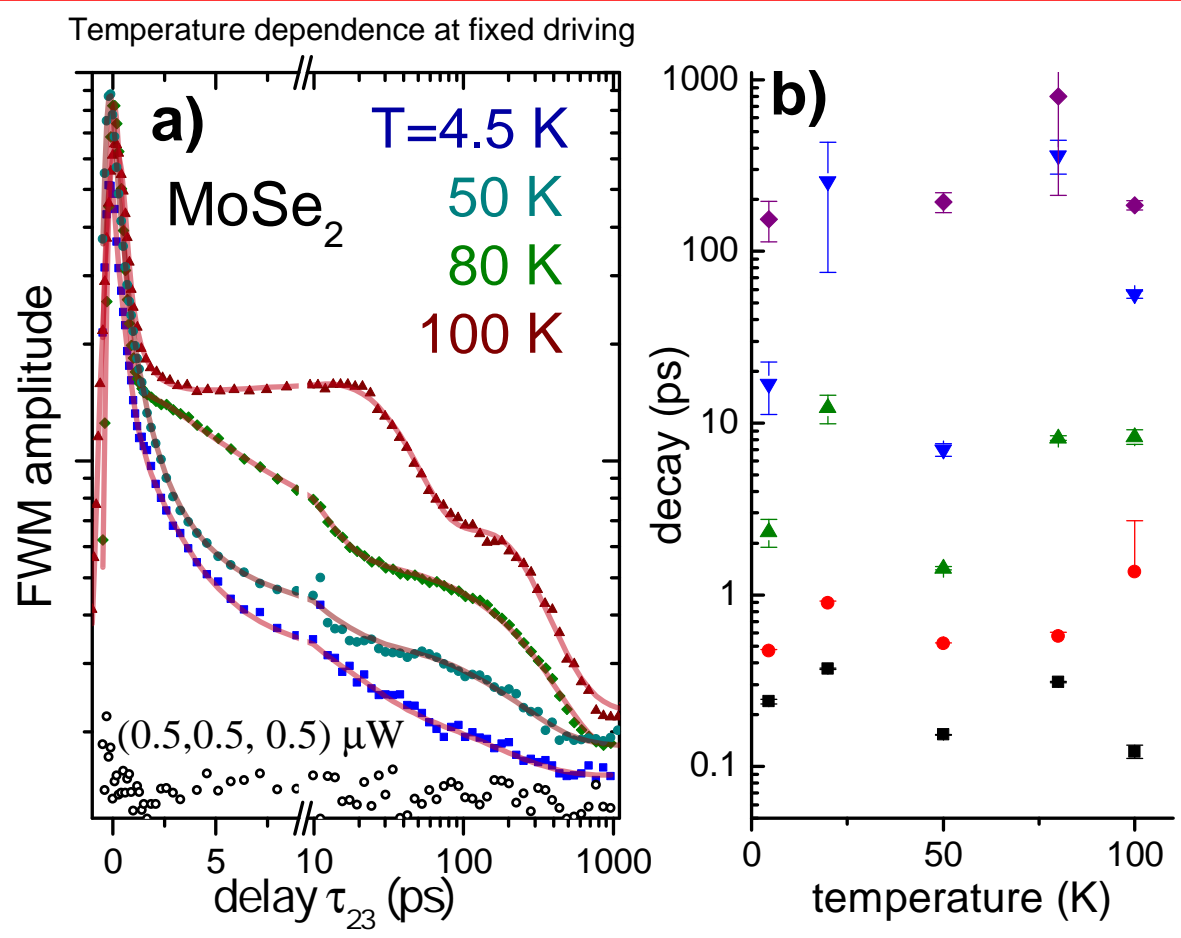

Figure 3. Exciton density dynamics in $\mathrm{MoSe}_{2}$ SL measured with four-wave mixing. Temperature dependence, as indicated, at fixed excitation power $P=0.5 \mu \mathrm{W}$. With increasing temperature unusual shapes of the delay traces are measured and reproduced by the modeling. In the fit we observe that the interference sets in when the components showing a longer decay increase their relative amplitudes.

refractive index dynamics. We expect that the phases are largely modified with increasing excitation powers, due to a larger population of dark states reservoir, consistently with our data.

To study the influence of phonon scattering and of thermal distribution across the states, we measure the EX density dynamics for increasing $T$ at fixed $P=0.5 \mu \mathrm{W}$, as shown in Fig. 3 a. Note that for each temperature the pulse wavelength was adjusted to match the EX transition, which was monitored with the reflectance of $\mathcal{E}_{1}$. Also, the temperature induced spatial drifts were corrected, such that the same sample spot was probed. The dynamics are fitted with $\left|R\left(\tau_{23}\right)\right|$ involving five decay components, explicitly given in Fig. $3 \mathrm{~b}$. For the fastest decay, we observe a decrease from around $300 \mathrm{fs}$ down to $100 \mathrm{fs}$, when increasing $T$ from $5 \mathrm{~K}$ to $100 \mathrm{~K}$. While no clear trends are observed for other decay components, the interplay of their relative amplitudes induces important changes in the FWM amplitude for $\tau_{23}>2 \mathrm{ps}$. For the lowest temperatures a monotonous decay of the FWM is observed when increasing $\tau_{23}$, as exemplified for $T=4.5 \mathrm{~K}$. From $T=50 \mathrm{~K}$ onward, a bump starts to build up at $\tau_{23} \simeq 100 \mathrm{ps}$. With increasing $T$, the bump progressively shifts to shorter $\tau_{23}$. At $T=100 \mathrm{~K}$, after the initial decay, the FWM amplitude increases, reaches maximum at $\tau_{23}=20 \mathrm{ps}$, to finally decay generating another bump at $\tau_{23}=200 \mathrm{ps}$. Again, such a surprising form of the $\tau_{23}$-delay dependence is due to the interference between different decay processes, building up when increasing $T$ owing to increasingly larger amplitude of processes exhibiting longer decay. This is a strong indication of increased population in a multiplicity of dark EX reservoirs with increasing temperature, mainly enabled by increased phonon scattering at higher temperatures.

\section{2 $\mathrm{WS}_{2}$ single layer}

We now discuss complementary results obtained on a $\mathrm{WS}_{2}$ SL. In Fig. 4 a we present the EX density dynamics measured at fixed $P=1.5 \mu \mathrm{W}$ for different temperatures. At low temperatures a strong pile up effect is observed. Namely, at $T=5 \mathrm{~K}$ after a dominating initial decay within $0<\tau_{23}<1 \mathrm{ps}$, a quasi-stationary signal with a minor modulation at around $100 \mathrm{ps}$ is observed, around an order of magnitude above the noise level. Interestingly, with 
Return to the Manage Active Submissions page at http://spie.org/submissions/tasks.aspx and approve or disapprove this submission. Your manuscript will not be published without this approval. Please contact author_help@spie.org with any questions or concerns.
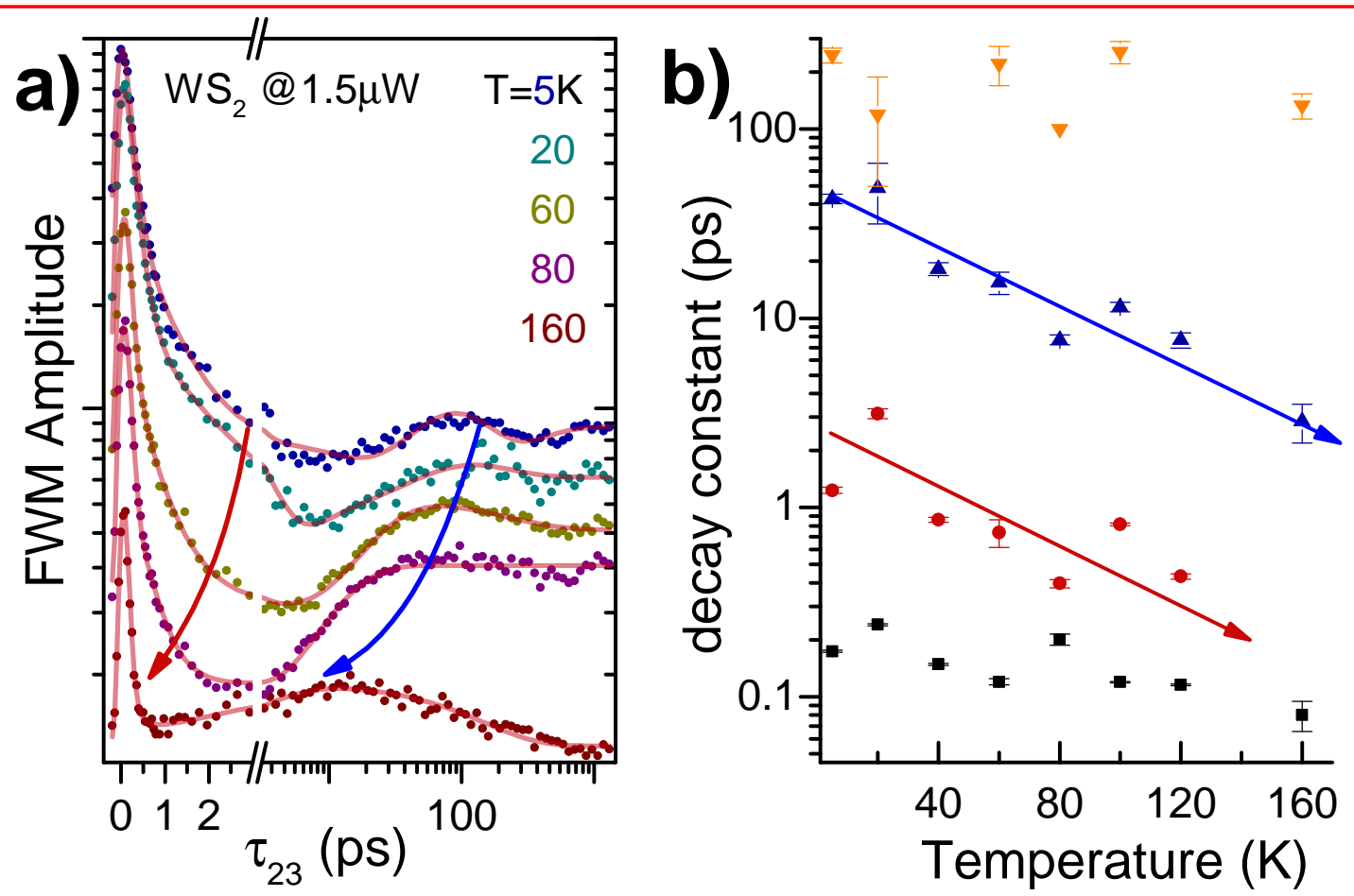

Figure 4. Exciton density dynamics in $\mathrm{WS}_{2}$ SL measured with four-wave mixing. Temperature dependence, as indicated, at fixed excitation power $P=1.5 \mu \mathrm{W}$. The modeling indicates shortening of the second and the third decay constants with increasing temperature. It is visible in the data as a shift of the bumps towards shorter delays $\tau_{23}$ when increasing the temperature.

increasing temperature, the initial loss of the signal becomes more pronounced, followed by the recovery, which here is most pronounced for $T=80 \mathrm{~K}$. At higher temperatures the recovery at longer $\tau_{23}$ is suppressed, such that a broad bump is visible at $T=160 \mathrm{~K}$. Qualitatively, we observe shifting of modulations to shorter delays with increasing $T$, as indicated with red and blue arrows.

The data are again modeled with the $\left|R\left(\tau_{23}\right)\right|$ function containing four components. The fitted decay constants are plotted in Fig. 4 b, from which we conclude that with increasing $T$ the dynamics strongly accelerates: when increasing $T$, values of $\tau_{1}, \tau_{2}$ and $\tau_{3}$ decrease. To interpret the data we remind that the bright EX in WS is located around $32 \mathrm{meV}$ above the optically inactive (spin-forbidden) one. The initial decay corresponds to the radiative decay and the scattering to dark states, also toward the optically inactive EX. The lower the temperature, the more efficiently EX population gets trapped in this state. In particular, at $T=5 \mathrm{~K}$ the Boltzmann factor $\exp (-32 \mathrm{meV} / 0.43 \mathrm{meV})=\exp (-74.4)$ entirely isolates the population from the thermal activation toward the bright EX. Alternative scattering channels are required to bring the population back to the bright EX state. Such a long relaxation time explains a very strong pile up effect observed at low temperatures. With increasing $T$ the thermal scattering from the spin-forbidden to spin-allowed EX state becomes relevant, for example at $T=160 \mathrm{~K}$ the Boltzmann factor rises to $\exp (-32 \mathrm{meV} / 13.7 \mathrm{meV}) \simeq 0.1$, enabling faster relaxation processes. This results in a faster decay and negligible pile-up at high temperatures, consistent with our findings.

Finally, we note that even at elevated temperatures, accumulation of the EX density for long $\tau_{23}$ and the pile up effect can be restored by increasing $P$ and thus occupancy of the dark EX states. This can be concluded from Fig. 5, showing intensity dependence of the FWM delay traces for different temperatures.

\section{CONCLUSIONS}

To conclude, using heterodyne FWM micro-spectroscopy, we measured temporal dynamics of EXs from 0.1 ps to $1.3 \mathrm{~ns}$ in $\mathrm{MoSe}_{2}$ and $\mathrm{WS}_{2}$ SLs after resonant excitation with a short laser pulse. The rich data set, including 
Return to the Manage Active Submissions page at http://spie.org/submissions/tasks.aspx and approve or disapprove this submission. Your manuscript will not be published without this approval. Please contact author_help@spie.org with any questions or concerns.

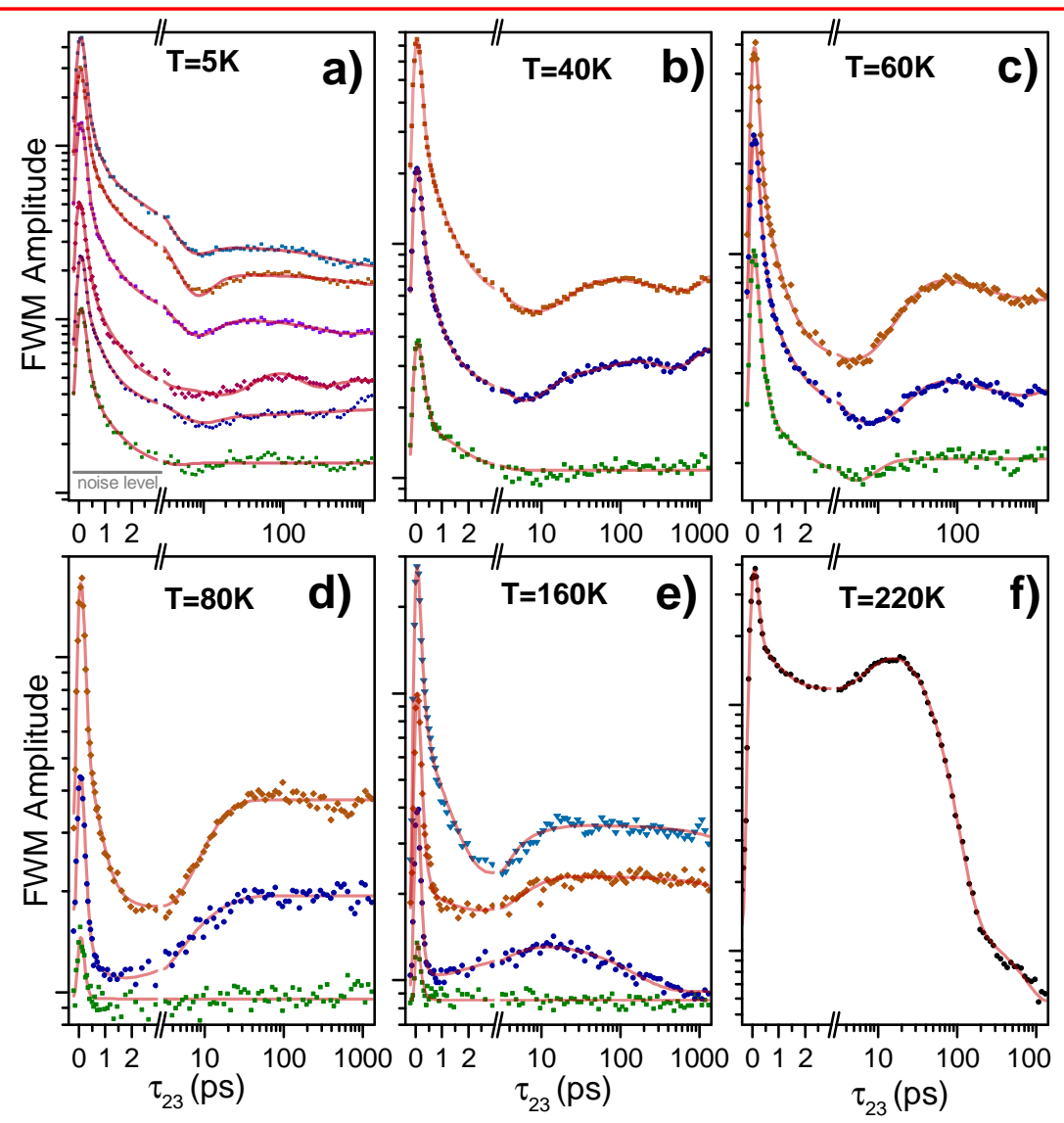

Figure 5. Exciton density dynamics in $\mathrm{WS}_{2} \mathrm{SL}$ measured with four-wave mixing. Power dependence measured at different temperatures as indicated. The used excitation powers are the following: a) $(0.2,0.4,0.8,2.7,5.2) \mu \mathrm{W}$, b) $(0.08,0.4,1.6) \mu \mathrm{W}, \mathrm{c})(0.08,0.4,1.5) \mu \mathrm{W}, \mathrm{d})(0.08,0.4,1.5) \mu \mathrm{W}, \mathrm{e})(0.4,1.5,3.6,7.5) \mu \mathrm{W}, \mathrm{f}) 6 \mu \mathrm{W}$.

excitation intensity and temperature dependence for both materials, is consistently modeled with a coherent superposition of several exponential decays. They are attributed to direct radiative decay, EX scattering out of the light cone, and spin-flip processes between spin-allowed and spin-forbidden EXs. Owing to the complex character of the data, unconventional interference effects arise in the FWM delay dependence, when different EX reservoirs display comparable occupancy. This permits to gain direct insight into interaction of bright excitons with the dark ones. As an outlook, we propose to use this approach to measure EX radiative lifetimes and evolution of EX dark states in van der Walls heterostructures with engineered thicknesses of encapsulating layers..$^{25}$

\section{REFERENCES}

[1] Borri, P., Langbein, W., Mørk, J., and Hvam, J. M., "Heterodyne pump-probe and four-wave mixing in semiconductor optical amplifiers using balanced lock-in detection," Opt. Express 169, 317 (1999).

[2] Dommers, S., Temnov, V. V., Woggon, U., Gomis, J., Martinez-Pastor, J., Laemmlin, M., and Bimberg, D., "Complete ground state gain recovery after ultrashort double pulses in quantum dot based semiconductor optical amplifiers," Appl. Phys. Lett. 90, 033508 (2007).

[3] Cesari, V., Borri, P., Rossetti, M., Fiore, A., and Langbein, W., "Refractive index dynamics and linewidth enhancement factor in p-doped InAs/GaAs quantum-dot amplifiers," IEEE J. Quantum Electron. 45, 579585 (2009).

[4] Langbein, W., Cesari, V., Masia, F., Krysa, A. B., Borri, P., and Smowton, P. M., "Ultrafast gain dynamics in InP quantum-dot optical amplifiers," Applied Physics Letters 97(21), 211103 (2010). 
Return to the Manage Active Submissions page at http://spie.org/submissions/tasks.aspx and approve or disapprove this submission. Your manuscript will not be published without this approval. Please contact author_help@spie.org with any questions or concerns.

[5] Masia, F., Accanto, N., Langbein, W., and Borri, P., "Spin-flip limited exciton dephasing in CdSe/ZnS colloidal quantum dots," Phys. Rev. Lett. 108, 087401 (2012).

[6] Naeem, A., Masia, F., Christodoulou, S., Moreels, I., Borri, P., and Langbein, W., "Giant exciton oscillator strength and radiatively limited dephasing in two-dimensional platelets," Phys. Rev. B 91, 121302 (2015).

[7] Becker, M. A., Scarpelli, L., Nedelcu, G., Raino, G., Masia, F., Borri, P., Stoferle, T., Kovalenko, M. V., Langbein, W., and Mahrt, R. F., "Long exciton dephasing time and coherent phonon coupling in $\mathrm{CsPbBr}_{2} \mathrm{Cl}$ perovskite nanocrystals," Nano Lett. 18(12), 7546-7551 (2018).

[8] Scarpelli, L., Masia, F., Alexeev, E. M., Withers, F., Tartakovskii, A. I., Novoselov, K. S., and Langbein, W., "Resonantly excited exciton dynamics in two-dimensional MoSe ${ }_{2}$ monolayers," Phys. Rev. B 96, 045407 (2017).

[9] Jakubczyk, T., Nayak, G., Scarpelli, L., Masia, F., Liu, W.-L., Dubey, S., Bendiab, N., Marty, L., Taniguchi, T., Watanabe, K., Coraux, G. N. J., Bouchiat, V., Langbein, W., Renard, J., and Kasprzak, J., "Coherence and density dynamics of excitons in a single-layer $\mathrm{MoS}_{2}$ reaching the homogeneous limit," ACS Nano 13, 3500-3511 (2019).

[10] Moody, G., Schaibley, J., and Xu, X., "Exciton dynamics in monolayer transition metal dichalcogenides," J. Opt. Soc. Am. B 33, C39 (2016).

[11] Robert, C., Lagarde, D., Cadiz, F., Wang, G., Lassagne, B., Amand, T., Balocchi, A., Renucci, P., Tongay, S., Urbaszek, B., and Marie, X., "Exciton radiative lifetime in transition metal dichalcogenide monolayers," Phys. Rev. B 93, 205423 (May 2016).

[12] Godde, T., Schmidt, D., Schmutzler, J., Aßmann, M., Debus, J., Withers, F., Alexeev, E. M., Del PozoZamudio, O., Skrypka, O. V., Novoselov, K. S., Bayer, M., and Tartakovskii, A. I., "Exciton and trion dynamics in atomically thin $\mathrm{MoSe}_{2}$ and $\mathrm{WSe}_{2}$ : Effect of localization," Phys. Rev. B 94, 165301 (Oct 2016).

[13] Plechinger, G., Nagler, P., Arora, A., Schmidt, R., Chernikov, A., Lupton, J., Bratschitsch, R., Schüller, C., and Korn, T., "Valley dynamics of excitons in monolayer dichalcogenides," Phys. Status Solidi RRL 11, 1700131 (2017).

[14] Langbein, W. and Patton, B., "Heterodyne spectral interferometry for multidimensional nonlinear spectroscopy of individual quantum systems," Opt. Lett. 31(8), 1151 (2006).

[15] Jakubczyk, T., Delmonte, V., Koperski, M., Nogajewski, K., Faugeras, C., Langbein, W., Potemski, M., and Kasprzak, J., "Radiatively limited dephasing and exciton dynamics in $\mathrm{MoSe}_{2}$ monolayers revealed with four-wave mixing microscopy," Nano Lett. 16, 5333-5339 (2016).

[16] Jakubczyk, T., Nogajewski, K., Molas, M. R., Bartos, M., Langbein, W., Potemski, M., and Kasprzak, J., "Impact of environment on dynamics of exciton complexes in a $\mathrm{WS}_{2}$ monolayer," 2D Mater. 5, 031007 (2018).

[17] Lu, Z., Rhodes, D., Li, Z., Tuan, D. V., Jiang, Y., Ludwig, J., Jiang, Z., Lian, Z., Shi, S.-F., Hone, J., Dery, H., and Smirnov, D., "Magnetic field mixing and splitting of bright and dark excitons in monolayer $\mathrm{MoSe}_{2}, "$ arXiv (2019).

[18] Dery, H. and Song, Y., "Polarization analysis of excitons in monolayer and bilayer transition-metal dichalcogenides," Phys. Rev. B 92, 125431 (2015).

[19] Moody, G., Kavir Dass, C., Hao, K., Chen, C.-H., Li, L.-J., Singh, A., Tran, K., Clark, G., Xu, X., Berghäuser, G., Malic, E., Knorr, A., and Li, X., "Intrinsic homogeneous linewidth and broadening mechanisms of excitons in monolayer transition metal dichalcogenides," Nat. Commun. 7, 8315 (2015).

[20] Kasprzak, J. and Langbein, W., "Coherent response of individual weakly confined exciton-biexciton systems," J. Opt. Soc. Am. B 29(7), 1766-1771 (2012).

[21] Kasprzak, J., Portolan, S., Rastelli, A., Wang, L., Plumhof, J. D., Schmidt, O. G., and Langbein, W., "Vectorial nonlinear coherent response of a strongly confined exciton-biexciton system," New J. Phys. 15, 055006 (2013).

[22] Mermillod, Q., Jakubczyk, T., Delmonte, V., Delga, A., Peinke, E., Gérard, J.-M., Claudon, J., and Kasprzak, J., "Harvesting, coupling, and control of single-exciton coherences in photonic waveguide antennas," Phys. Rev. Lett. 116, 163903 (2016). 
Return to the Manage Active Submissions page at http://spie.org/submissions/tasks.aspx and approve or disapprove this submission. Your manuscript will not be published without this approval. Please contact author_help@spie.org with any questions or concerns.

[23] Delmonte, V., Specht, J. F., Jakubczyk, T., Höfling, S., Kamp, M., Schneider, C., Langbein, W., Nogues, G., Richter, M., and Kasprzak, J., "Coherent coupling of individual quantum dots measured with phasereferenced two-dimensional spectroscopy: Photon echo versus double quantum coherence," Phys. Rev. B 96, 041124 (Jul 2017).

[24] Perea-Causín, R. 1., Brem, S., Rosati, R., Jago, R., Kulig, M., Ziegler, J. D., Zipfel, J., Chernikov, A., and Malic, E., "Exciton propagation and halo formation in two-dimensional materials," Nano Letters 19(10), 7317-7323 (2019).

[25] Fang, H. H., Han, B., Robert, C., Semina, M. A., Lagarde, D., Courtade, E., Taniguchi, T., Watanabe, K., Amand, T., Urbaszek, B., Glazov, M. M., and Marie, X., "Control of the exciton radiative lifetime in van der waals heterostructures," Phys. Rev. Lett. 123, 067401 (2019). 